\title{
IDDES Evaluation of Oscillating Hydraulic Jumps
}

\author{
Vimaldoss Jesudhas ${ }^{1, *}$, Frédéric Murzyn ${ }^{2}$, and Ram Balachandar ${ }^{1}$ \\ ${ }^{1}$ Department of Civil and Environmental Engineering, University of Windsor, Ontario, Canada. \\ ${ }^{2}$ ESTACA West Campus, Department of Mechanical Engineering, 53000 Laval, France
}

\begin{abstract}
This paper presents the results of three-dimensional, unsteady, Improved Delayed Detached Eddy Simulations of an oscillating and a stable hydraulic jump at Froude numbers of 3.8 and 8.5, respectively. The different types of oscillations characterised in a hydraulic jump are analysed by evaluating the instantaneous flow field. The instability caused by the flapping wall-jet type flow in an oscillating jump is distinct compared to the jump-toe fluctuations caused by the spanwise vortices in the shear layer of a stable jump. These flow features are accurately captured by the simulations and are presented with pertinent discussions. The near-bed vortical structures in an oscillating jump is extracted and analysed using the $\lambda_{2}$ criterion.
\end{abstract}

\section{Introduction}

The transition from a supercritical to a subcritical open-channel flow is termed as a hydraulic jump. They occur in rivers and streams and are often artificially created to dissipate energy in hydraulic structures. They are characterised by strong turbulence, violent fluctuations of the free surface and air entrainment. Due to their practical significance, several experimental studies [1-7] have been aimed at understanding the complex flow features. However, these studies have been only partially successful due to the limitations of the measuring techniques in air-water multiphase flows. Moreover, experiments probe a point or a plane, the complete three-dimensional (3D) flow field is not available for analysis. Recent improvements in computing capabilities have rendered numerical simulation as a viable tool to analyse the hydraulic jump flow field [8-14].

Hydraulic jumps can be classified into five types based on the Froude number $F_{1}=U_{1} / \sqrt{g d_{1}}$ [2]. Here, $U_{1}$ and $d_{1}$ are the supercritical velocity and flow depth, respectively, and $g$ is the acceleration due to gravity. The classification is as follows: (i) undular jump $\left(1<F_{1}<1.7\right)$ (ii) weak jump $\left(1.7<F_{1}<2.5\right)$ (iii) oscillating jump $\left(2.5<F_{1}<\right.$ $4.5)$ (iv) stable jump $\left(4.5<F_{1}<9\right)$ and (v) rough jumps $\left(F_{1}>9\right)$. The schematic of the typical flow field in an oscillating and a stable jump is presented in Figs. 1a and 1b. The instability in the oscillating jump is caused by the flapping of the wall-jet flow as depicted in Fig. 1a. The jump roller is weak compared to the stable jump and occupies a small region close to the free surface. The flapping of the wall-jet generates waves that propagate downstream. The

\footnotetext{
* Corresponding author: jesudha@uwindsor.ca
} 
stable jump has a well-defined roller with the wall-jet region below it (Fig. 1b). However, the jump toe of the stable jump oscillates about a mean position due to counter-rotating horizontal vortices formed in the shear layer between the roller and the wall-jet flow $[14,15]$. Researchers have attempted to characterize the different types of oscillations using qualitative techniques (high-speed photography) or by identifying the dominant frequencies in the flow $[15,16]$. However, a systematic evaluation of the oscillating characteristics by assessing the complete 3D flow field has not been achieved. To address this limitation, an oscillating jump (OJ) and a stable jump (SJ) at Froude numbers 3.8 and 8.5 are simulated using unsteady, 3D, Improved Delayed Detached Eddy Simulation (IDDES). Volume of Fluid (VOF) coupled with High-resolution Interface Capturing (HRIC) technique was used to capture the free surface. The present paper presents the results of these simulations, showcasing the potential of CFD analysis to characterise the oscillating nature of hydraulic jumps. The mean flow field predicted by the simulations is presented with relevant analysis. The different types of oscillations in hydraulic jumps are evaluated by examining the instantaneous flow field. The near-bed vortical structures present in an oscillating jump is identified using the $\lambda_{2}$ criterion [17].

(a) Oscillating Jump (OJ)

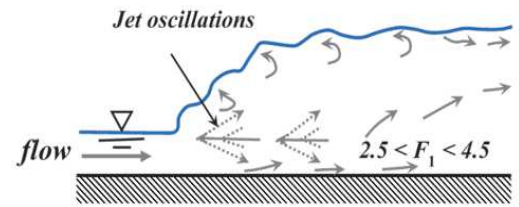

(b) Stable Jump (SJ)

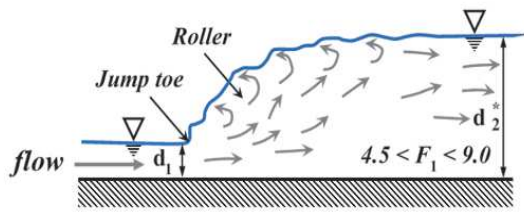

(c)

Top (symmetry)

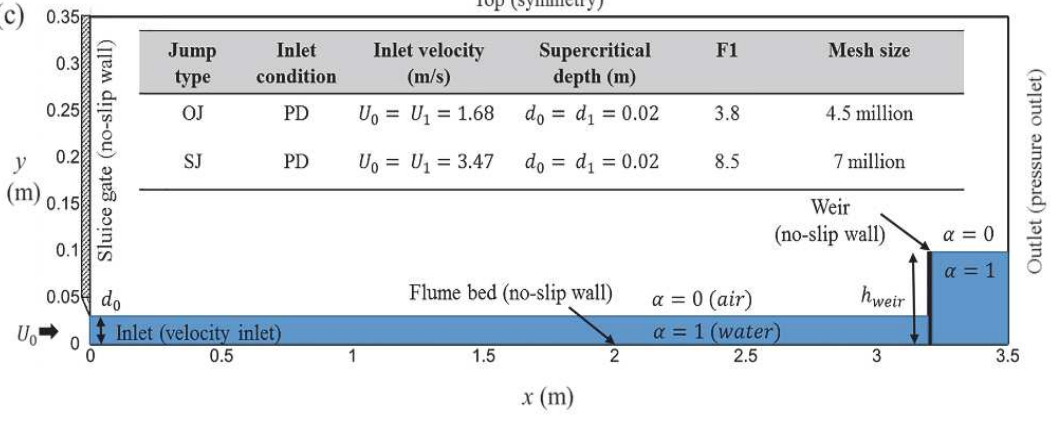

Fig. 1. Types of hydraulic jumps (a) Oscillating Jump (OJ), (b) Stable Jump (SJ); (c) 2D representation of the $3 \mathrm{D}$ computational domain for the $\mathrm{SJ}$ simulation at time $t=0$

\section{Computational Setup}

Most of the earlier simulations on hydraulic jumps have been performed using ReynoldsReynolds-Averaged Navier-Stokes (RANS) models [8-11]. Ma et al., [12] compared the performance of RANS and Detached-Eddy simulations (DES) in simulating hydraulic jumps. He reported that the DES model was superior than RANS in resolving the free surface features of hydraulic jumps. IDDES model is an improved version of DES proposed by [18]. Similar to DES, it uses a hybrid RANS - Large Eddy Simulation (LES) approach to model turbulence. The blending function used to switch between the RANS and LES regions is not only dependent on the grid size but also on the wall normal distance, thereby avoiding the 
"log-law" mismatch error of DES. Unlike the RANS, IDDES can resolve the anisotropic structures in the flow and is also computationally less exhaustive than the LES approach. The complete formulation of the model is available in [18-20]. The two-dimensional (2D) representation of the $3 \mathrm{D}$ computational domain used in the simulation of the $\mathrm{SJ}\left(F_{1}=8.5\right)$ is shown in Fig. $1 \mathrm{c}$ at time $t=0$. The dimensions of the domain used for the SJ simulation was $3.5 \mathrm{~m} \times 0.25 \mathrm{~m} \times 0.35 \mathrm{~m}$. While other dimensions remained the same, a lower domain height was used to simulate the $\mathrm{OJ}\left(F_{1}=3.8\right)$, thereby reducing the overall mesh size. Hexahedral mesh was used in the simulations. The grid size was identified based on a grid dependency study. Prism layers were used near the walls with grid refinements in the regions of interest. Due to the varying nature of $y^{+}$, all- $y^{+}$wall treatment available in STAR-CCM+ was employed to capture the wall effects. The boundary conditions employed is also presented in Fig. 1c. The initial volume fraction $\alpha$, was set as shown in Fig. 1c to reduce the computation time. No-slip condition was applied to the side walls. IDDES uses a blending function to switch between RANS and LES. This function was monitored to ensure that LES was performed in our regions of interest. The time-step used was $1 \mathrm{~ms}$, the solution was averaged for $10 s$ for computing the mean quantities.

\section{Validation}

CFD can play an important role in filling our gaps in knowledge when it is difficult to accurately measure important flow parameters by providing a detailed picture of the entire flow field. However, an inappropriate numerical model will undoubtedly produce erroneous results. To address this, the numerical model was validated based on the standardized procedures documented in AIAA Guide for Verification and Validation of Computational Fluid Dynamics Simulations [21]. All mean and turbulent flow features commonly reported in hydraulic jump literature was validated with several experimental data. This exhaustive validation procedure is documented in $[13,14]$. As a sample, Fig. 2a shows the comparison of normalized mean total pressure $p /\left(0.5 \rho_{w} U_{1}^{2}\right)$, where $\rho_{w}$ is the density of water, with the experimental results of [6] at two streamwise locations. The profiles of mean total pressure predicted by the simulation agree well with the experimental data at both locations with a difference of less than $10 \%$, which is typical in the hydraulic jump flow field [11]. Wang [7] showed that the decay of mean air concentration can be collapsed onto a single line by using the roller length $L_{r}$, as the horizontal length scale. This plot is shown in Fig. 2b, in which are also plotted the experimental results of $[5,7]$ for the same Froude number. The mean air concentrations predicted by the simulation agree well with the experimental data up to $60 \%$ of the roller length. Beyond this the numerical simulations predict a slightly higher air concentration than the experimental results, which can be attributed to the numerical diffusion of the VOF model. On establishing confidence in the simulation results, the flow field predicted by the IDDES were further analysed.

\section{Results and Discussions}

Figures $3 \mathrm{a}$ and $3 \mathrm{~b}$ show the mean free surface predicted by the simulations for the $\mathrm{OJ}\left(F_{1}=\right.$ $3.8)$ and $\mathrm{SJ}\left(F_{1}=8.5\right)$, respectively. The jump toe region (dashed circles in Figs. 3a and $3 \mathrm{~b}$ ) can be identified by the steep rise in free surface height. Between the two jumps, this location varies in the spanwise direction [16]. Deformations in the free surface are also visible in the figures. Typical instantaneous free surface is presented in Figs. 3c and 3d, respectively for the two jumps. Intense undulations of the free surface can be observed in the figures. It can be visually observed that the intensity of the free-surface undulations is higher as the Froude number increases [5]. Compared to the mean flow field, the instantaneous location of the 
jump toe for the SJ (Fig. 3d) is considerably upstream compared to the mean location seen in Fig. 3b. This is due to the jump toe oscillations.

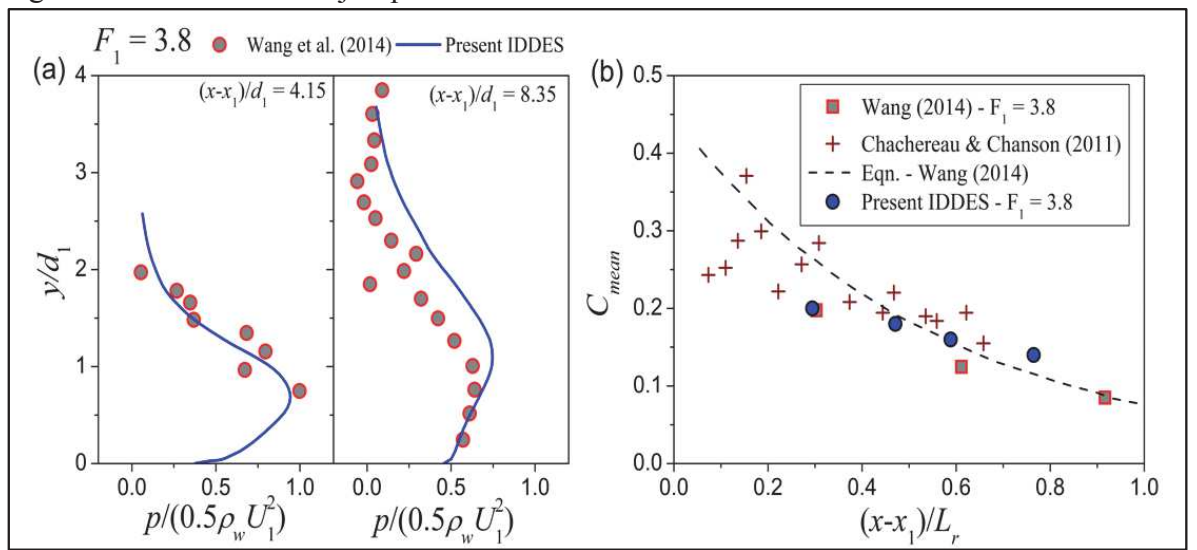

Fig. 2. Validation of CFD results (a) Mean total pressure comparison at two streamwise locations of OJ (b) Decay of air concentration within the jump roller

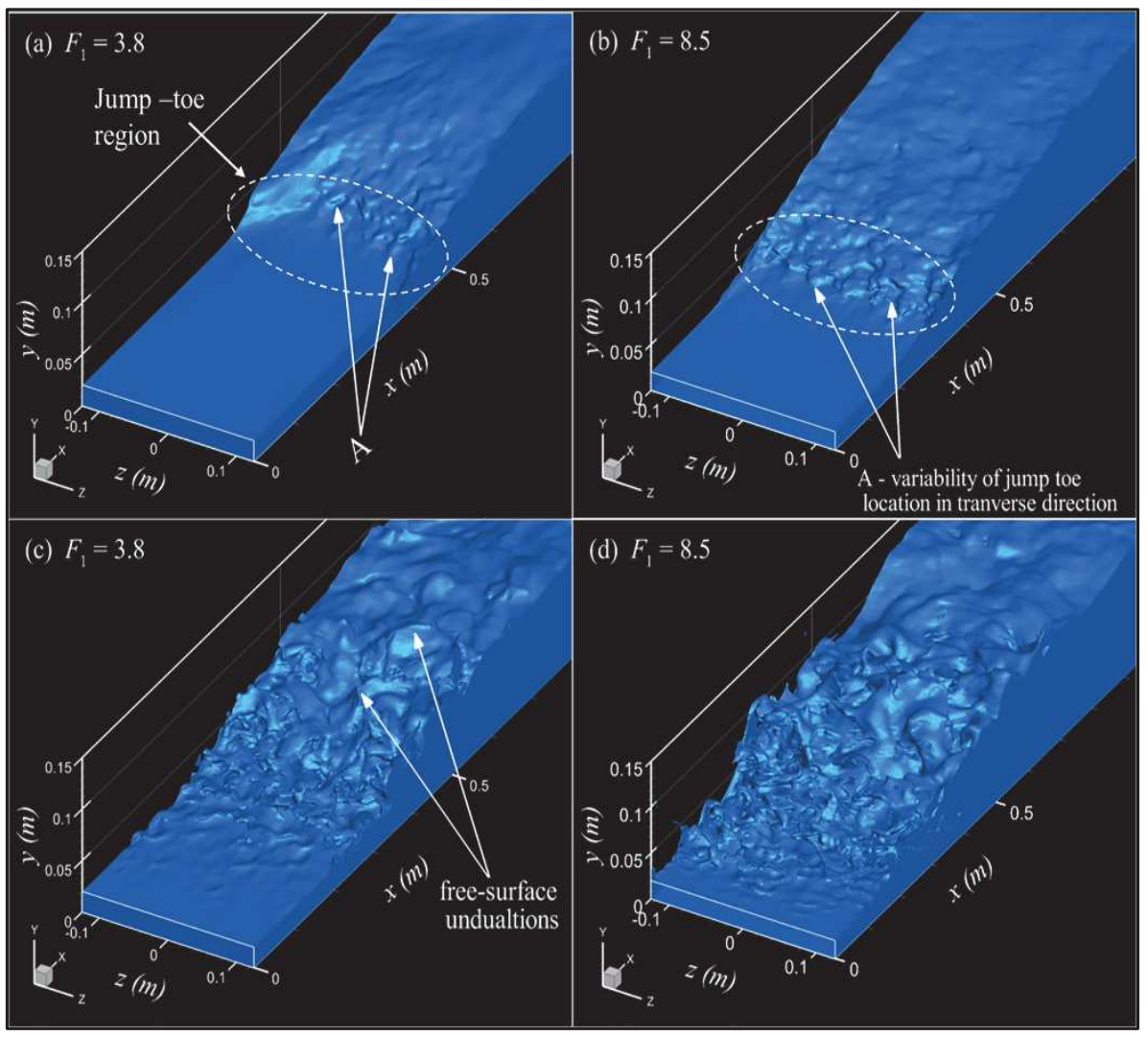

Fig. 3. Mean free surface: (a) OJ, (b) SJ; Instantaneous free surface: (c) OJ, (d) SJ 
Figure 4a depicts the contours of the mean streamwise velocity in the central plane of the OJ. The mean velocity vectors are also superimposed in Fig.4a. The OJ has a very weak roller as evidenced by the small negative flow region close to the free surface. In the absence of a strong roller, the wall jet type flow emanating from the sluice gate detaches from the wall due to the adverse pressure gradient and flaps in the vertical direction. This vertical flapping of the wall-jet flow generate waves that propagate in the downstream direction. The wave like motion generates near-zero mean velocity in the bed region (marked by the dashed circle in Fig. 4a). A shear layer is formed between the roller and the wall jet flow. As expected, the Reynolds stress $\overline{u^{\prime} v^{\prime}} / U_{1}^{2}$, where $u^{\prime}$ and $v^{\prime}$ are the streamwise and vertical velocity fluctuations, is maximum in this region as shown in Fig. 4b. Fig. 4c shows the contours of mean streamwise velocity superimposed by the velocity vectors in the central plane of the SJ. A strong roller region is clearly visible in Fig.4c with the wall jet flow below it. As in a OJ, a shear layer is formed between the roller and the wall jet flow causing the Reynolds stresses to be maximum in this region (Fig. 4d). Unlike the OJ, the wall jet flow in $\mathrm{SJ}$ adheres to the bed due to its higher momentum and the presence of the strong roller above it.
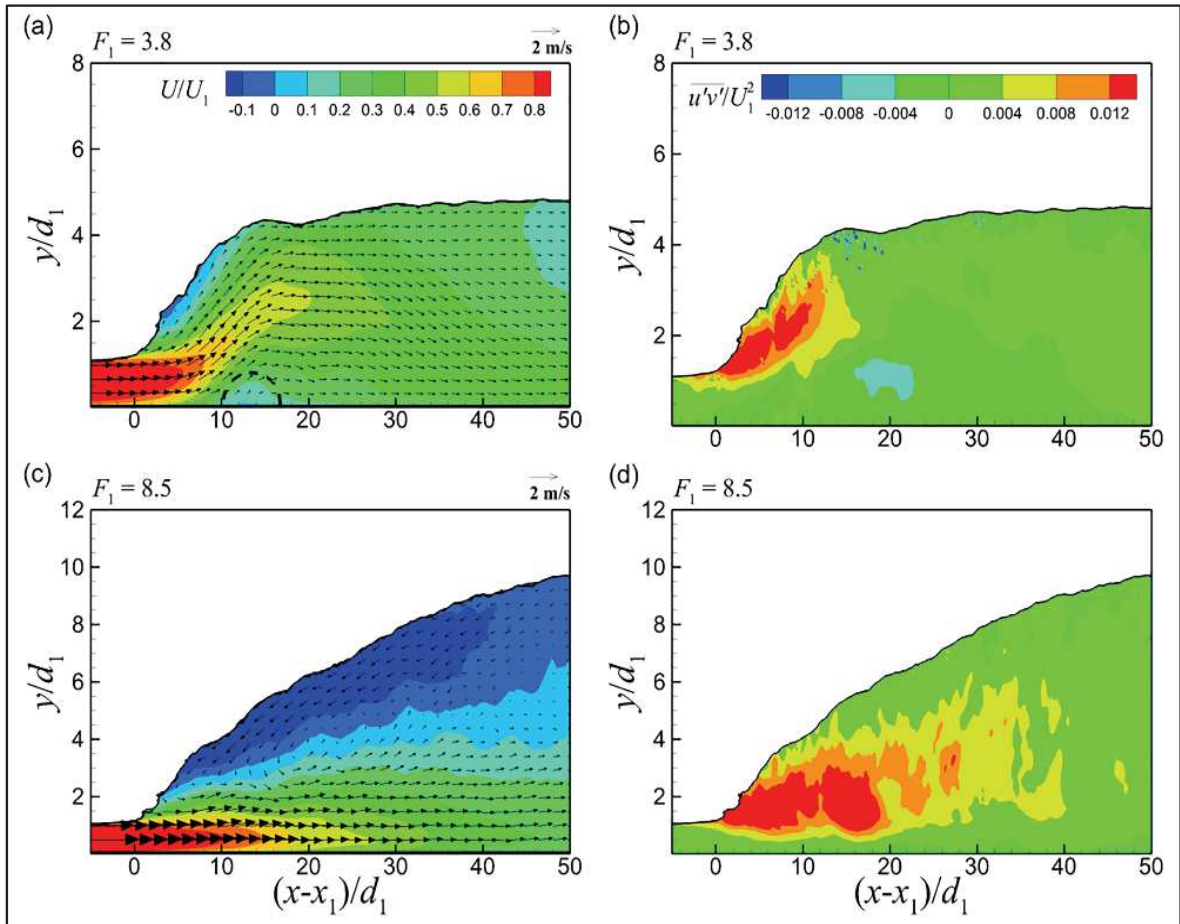

Fig. 4. Contours of (a) mean streamwise velocity superimposed by mean velocity vectors and (b) Reynolds shear stress in the central plane of OJ; Contours of (c) mean stream wise velocity superimposed by mean velocity vectors and (d) Reynolds shear stress in the central plane of SJ.

Figures $5 \mathrm{a}$ and $5 \mathrm{~b}$ depict the contours of instantaneous streamwise velocity $u$, superimposed by the instantaneous velocity vectors at two different time instances ( $t=35.5$ and $36 s$ ) for the OJ. The vertical flapping of the wall jet can be observed at these time instances. The resulting wave like flow causes reverse flow regions close to the bed (marked by dashed circles in Figs. 5a and 5b). The reverse flow region at $t=35.5 \mathrm{~s}$ is presented as an inset in Fig. 5a. These near-bed reverse flow regions can cause pressure fluctuations. This is 
the reason a SJ is preferred for energy dissipation applications in hydraulic structures. Pockets of air enter the flow at the jump toe as marked in Fig. 5b. This phenomenon is termed as local aeration and is caused by the impact of the jump roller on the emanating wall jet. The presence of horizontal vortices in the shear layer that advect the air pockets downstream [15, 16]. However, since the roller is weak, such organized horizontal vortical structures was not captured in the $\mathrm{OJ}$ at these time instances. The contours of instantaneous streamwise velocity superimposed by the instantaneous velocity vectors at two instances $(t=35.5$ and $36 s)$ for the SJ are shown in Figs. 5c and 5d. The wall-jet flow adheres to the wall at all time instances, with a strong roller above it. Pockets of air (marked in Fig. 5d) enter the flow at the jump toe due to local aeration. The vectors show the formation of counter-rotating horizontal vortices (marked in Fig. 5c) in the shear layer that advect the air pockets further downstream. These horizontal vortices (marked in Fig. 5c) are responsible for the jump toe oscillations in the SJ which is distinctive compared to the jet-flapping oscillations observed in an OJ.

To further investigate the vortical structures in the OJ, the instantaneous $z$-vorticity is plotted at two different time instances $(t=35.5$ and $36 s)$ in Figs. 6a and 6b. Strong $z$-vortical structures (red color in Figs. 6a and 6b) are formed in the shear layer between the roller and the flapping wall jet. The wave like pattern and the resulting reverse flow regions cause these vortical structures to interact with the counter-rotating near-bed $z$-vortical structures (blue color in Figs. 6a and 6b) causing them to detach and advect downstream. These near-bed vortical structures are captured using $\lambda_{2}$ criteria [17] and presented in Fig. 6c. Tube like organized structures are present in the near-bed region of the OJ. The dynamics of these structures must be further evaluated to understand the air-water dynamics in OJ.
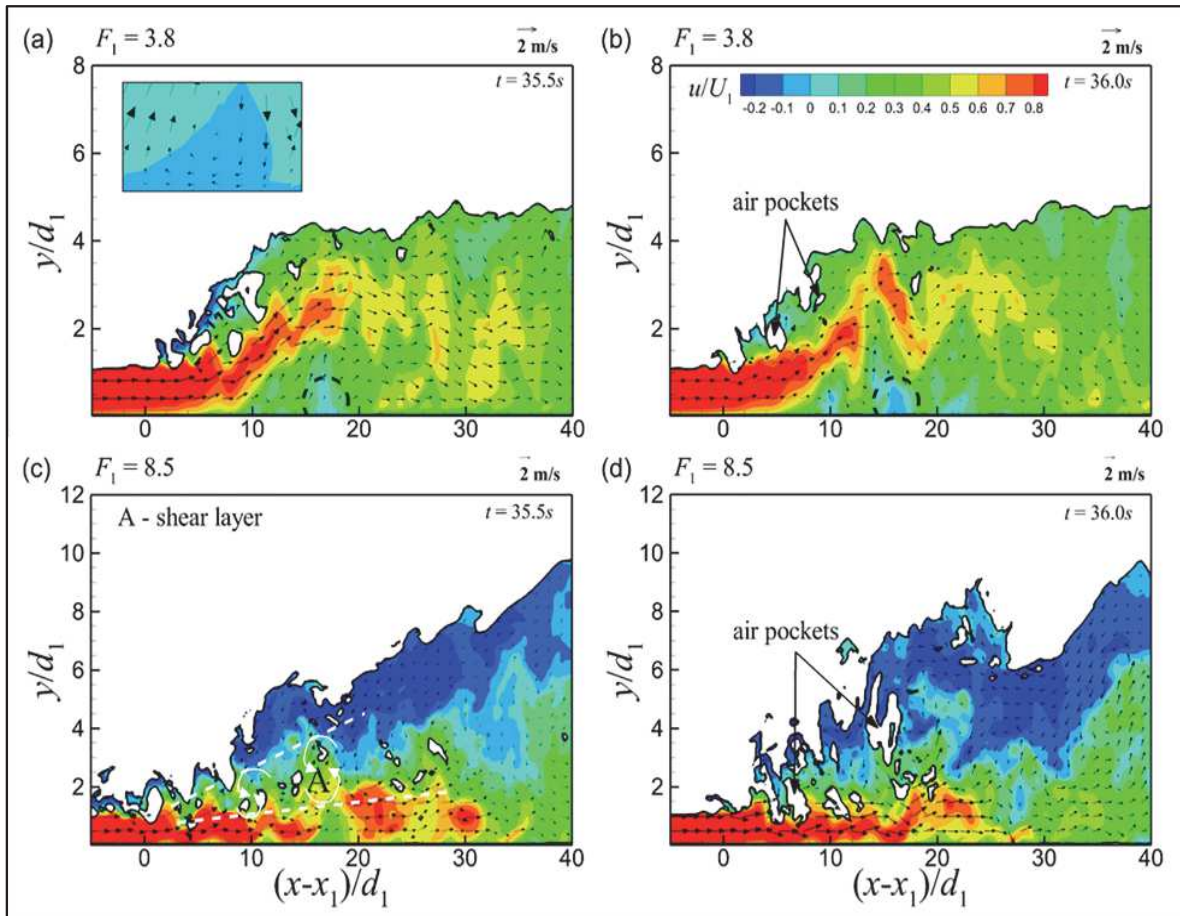

Fig. 5. (a-b) Contours of instantaneous streamwise velocity superimposed by velocity vectors at two time instances at the central plane of OJ; (c-d) Contours of instantaneous streamwise velocity superimposed by velocity vectors at two time instances at the central plane of SJ. 


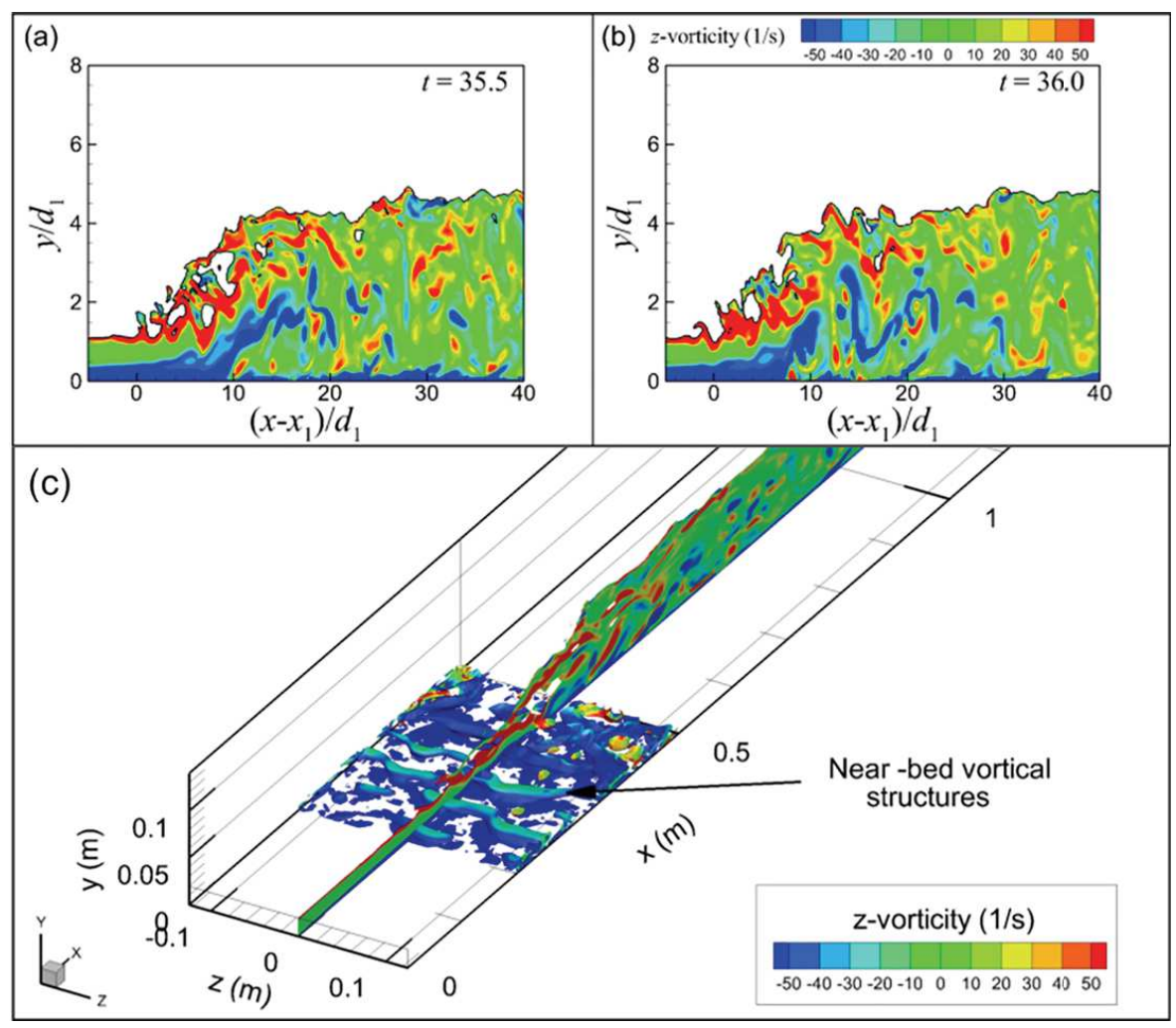

Fig. 6. (a-b) Typical instantaneous z-vorticity contours in the central plane of OJ $\left(F_{1}=3.8\right)$ at two time instances (c) Near-bed vortical structure in OJ captured using $\lambda_{2}$ criteria.

\section{Conclusions}

Three-dimensional, unsteady, Improved Delayed Detached Eddy Simulation (IDDES) was used to model an oscillating and a stable hydraulic jump. Volume of Fluid (VOF) with High Resolution Interface Capturing (HRIC) was used to capture the air-water multiphase flow. The results showed that the simulations were accurate in capturing the complex flow physics of hydraulic jumps. The instabilities caused by the flapping jet in an oscillating jump was accurately modelled by the simulations. The near-bed vortical structures that were generated by the wave like motion in an oscillating jump was educed using $\lambda_{2}$ criteria. Horizontal vortices that are present in the shear layer of a stable jump advect the entrained air downstream. These vortices are also responsible for the jump-toe oscillations in a stable jump, which is distinctive from the jet flapping oscillations in an oscillating jump.

\section{References}

1. Rajaratnam, N., Advances in hydroscience, 4, 197-280 (1967)

2. Hager, W.H., Energy Dissipators and Hydraulic Jump (Kluwer Academic Publishers, Water Science and Technology Library, Dordrecht, The Netherlands, 1982). 
3. Chanson, H., Brattberg, T., International Journal of Multiphase Flow, 26(4), 583$607(2000)$

4. Murzyn, F., Mouaze, D., Chaplin, J.R., International Journal of Multiphase Flow, 31(1), 141-154 (2007)

5. Chachereau, Y., Chanson, H., Experimental Thermal and Fluid Science, 35(6), 896909 (2011)

6. Wang, H., Murzyn, F., Chanson, H., Experiments in fluids, 55(11), p.1847 (2014)

7. Wang, H., Turbulence and Air Entrainment in Hydraulic Jumps, (Doctoral dissertation, The University of Queensland, Brisbane, Australia, 2014)

8. Long, D., Steffler, P. M., Rajaratnam, N., Journal of Hydraulic Research, 29(3), 293-308 (1991)

9. Chippada, S., Ramaswamy, B., Wheeler, M. F., International Journal for Numerical Methods in Engineering, 37(8), 1381-1397 (1994)

10. Ma, F., Hou, Y., Prinos, P., Journal of hydraulic research, 39(5), 493-503 (2001)

11. Ma, J., Oberai, A.A., Lahey, R.T., Drew, D.A., Heat and mass transfer, 47(8), p.911 (2011)

12. Witt, A., Gulliver, J., Shen, L., International Journal of Multiphase Flow, 72, 165 180 (2015)

13. Jesudhas, V., Roussinova, V., Balachandar, R., Barron, R., Journal of Hydraulic Engineering, 143(3), (2016)

14. Jesudhas, V., Balachandar, R., Roussinova, V., Barron, R., Journal of Hydraulic Engineering, 144(6), 04018022 (2018).

15. Mossa, M., Journal of Hydraulic Research, 37(4), 541-558 (1999)

16. Zhang, G., Wang, H., Chanson, H., Environmental fluid mechanics, 13(2), 189-204 (2013)

17. Jeong, J., Hussain, F., Journal of fluid mechanics, 285, 69-94 (1995)

18. Shur, M.L., Spalart, P.R., Strelets, M.K., Travin, A.K., International Journal of Heat and Fluid Flow, 29(6), 1638-1649 (2008)

19. Jesudhas, V., Modeling of free-surface flows with air entrainment (Doctoral dissertation, University of Windsor, Canada, 2016).

20. STAR-CCM+, User Guide (CD-adapco, v8.06, 2013)

21. AIAA Standards Committee, AIAA Guide for the Verification and Validation of Computational Fluid Dynamics Simulations (G-077-1998, 2002) 